\title{
Cognitive Radio Techniques for Satellite Communication Systems
}

\author{
(Invited Paper) \\ Shree Krishna Sharma, Symeon Chatzinotas, and Björn Ottersten \\ SnT - securityandtrust.lu, University of Luxembourg \\ Email:\{shree.sharma, symeon.chatzinotas, bjorn.ottersten\}@uni.lu
}

\begin{abstract}
The usable satellite spectrum is becoming scarce due to continuously increasing demand for broadcast, multimedia and interactive services. In this context, cognitive satellite communications has received important attention lately in the research community. Exploring efficient spectrum sharing techniques for enhancing spectral efficiency in satellite communication has become an important research challenge. In this paper, we study the main aspects of satellite cognitive communications and present possible practical scenarios for hybrid/dual cognitive satellite systems. Furthermore, suitable cognitive techniques for the considered scenarios are identified. More specifically, Spectrum Sensing (SS), interference modeling, and beamforming techniques are discussed for hybrid cognitive scenario and SS, interference alignment, and cognitive beamhopping techniques are discussed for dual satellite systems. This paper concludes by providing interesting open research issues in this domain.

Index Terms-Cognitive Satellite Communications, Spectrum Sensing, Underlay, Dual Satellite Systems
\end{abstract}

\section{INTRODUCTION}

The demand for broadband wireless spectrum is increasing due to a rapidly expanding market of high speed broadband and multimedia wireless services while the usable spectrum is becoming scarce due to current spectrum segmentation and static frequency allocation policies. However, different spectrum occupancy measurement campaigns carried out at different parts of the world show that the average spectrum occupancy rate is very low [1] and it shows spatial as well as temporal variations. In this context, Cognitive Radio (CR) communication can be an efficient technique to enhance the spectrum efficiency in the context of coexistence of heterogeneous wireless networks. Wireless networks may exist within the same spectrum band in different ways such as two terrestrial networks or two satellite networks or satelliteterrestrial hybrid networks. Cognitive techniques allow the coexistence of primary and secondary systems within the same spectrum without obstructing the normal operation of the primary licensed systems.

In the context of Satellite Communications (SatComs), Fixed Satellite Services (FSS) commonly use C and K band frequencies and for Mobile Satellite Services (MSS), L and S frequency bands are better suited since they permit small onboard antennas due to better foliage penetration and smaller impact of atmospheric affects. Furthermore, there has been continued pressure on satellite bands, especially in L and C bands due to the introduction of new terrestrial services such as 3G mobile telephony, LTE, WiMax and WiFi services. Due to high demand of broadband services and limited availability of L and S-band frequency resources, higher frequency bands i.e., $\mathrm{Ku}$ and $\mathrm{Ka}$ bands have also been assigned for MSS systems [2]. At present, Ku band based MSS systems are able to provide broadband services to many vehicular users such as trains, boats, planes, and cars. In this context, exploring efficient frequency sharing techniques to enhance spectral efficiency while guaranteeing quality of service is a highly relevant and challenging research problem. This has led to the concept of cognitive SatComs which exploits the opportunities for spectrum sharing between two satellite systems or between satellite and terrestrial systems [3].

The most common cognitive techniques in the literature can be categorized into interweave or Spectrum Sensing (SS), underlay, overlay and Database (DB) related techniques. In SS only techniques, Secondary Users (SUs) are allowed to transmit whenever Primary Users (PUs) do not use a specific band, whereas in underlay techniques, SUs are allowed to transmit as long as they meet the interference constraint of the PUs. Overlay networks are characterized by the mitigation of interference with the help of advanced coding and transmission strategies at the cognitive transmitters while in the DB technique, cognitive terminals query the predefined DB in order to find the unoccupied frequency bands and transmit in these bands. From practical perspectives, the aforementioned techniques differ in terms of operational requirements and implementation complexities.

The following features are unique to cognitive SatComs and can be considered while investigating satellite CR techniques [3].

- Polarization and elevation angle can be considered as additional degrees of freedom for enabling the coexistence of satellite and terrestrial networks.

- The satellite uplink transmissions with low elevation angles tend to receive more interference from terrestrial systems compared to the uplinks with higher elevation angles.

- The power in the space segment is quite limited and there already exists some level of inter-satellite and/or intrasatellite interference.

- Implementing dynamic SS in satellite downlink may be difficult due to large coverage areas and weak signal levels even in case of multispot coverage. 
- Wideband sensing techniques are required for detecting $\mathrm{Ku} / \mathrm{Ka}$ band satellite signals and several narrowband SS techniques investigated in the context of terrestrial CR may not be applicable.

- The resource management problem in satellite CR networks differs from the one in isolated wireless systems since resources are not uniform and they may not belong to the same operator.

- The directional characteristics of fixed satellite terminals can be exploited while investigating suitable coexistence techniques.

The CR research is more mature in the terrestrial context but has received limited attention in the satellite context. In the field of satellite communications, the main available literature related to satellite-terrestrial coexistence has considered hybrid or integrated satellite networks [4], [5]. The available CR related satellite literature can be broadly categorized into (i) hybrid cognitive SatComs [3], [6]-[13], and (ii) dual cognitive SatComs [14]-[18]. In this paper, we present possible coexistence scenarios for hybrid and dual cognitive SatComs. Furthermore, we identify suitable cognitive techniques for these scenarios and discuss their applicability in the context of cognitive SatComs. Moreover, we provide interesting open research issues in this domain.

\section{Hybrid Cognitive SatComs}

\section{A. Scenarios}

This scenario deals with the coexistence of satellite and terrestrial links within the same spectrum for enhancing the overall spectral efficiency. The communication links can be i) from satellite to satellite terminals, ii) from terrestrial BS to terrestrial user terminals, iii) from satellite terminals to the satellite, iv) from terrestrial user terminals to the terrestrial BS. While considering possible combinations of these links, the following four different coexistence modes are possible [3]: i) Forward Normal mode, ii) Forward Reverse Mode, iii) Return Normal Mode, and iv) Return Reverse Mode. In this context, two priority conditions can be set i.e., by providing primary access to the satellite or to the terrestrial link. In many cases, satellite link can be considered as primary link due to adverse transmission conditions and in some of the cases, terrestrial link can be considered as primary. The latter case is beneficial for satellite operators to take advantage of unused terrestrial spectrum to maximize their network capacity.

The hybrid scenario may comprise of a multi-user link coexistence (Fig. 1) and a single-user link coexistence (Fig. 2). In the multiuser link coexistence scenario, $\mathrm{S} / \mathrm{C}$ band multibeam satellite systems can coexist with terrestrial cellular systems such as WiMax, LTE using suitable cognitive techniques. Suitable cognitive techniques allow the reuse of the satellite licensed spectrum in the terrestrial secondary systems or vice versa without disturbing the operation of the licensed primary systems. In the single-user link coexistence scenario, Ka band FSS satellite systems can coexist with the microwave Fixed Service (FS) links by exploiting suitable cognitive techniques.

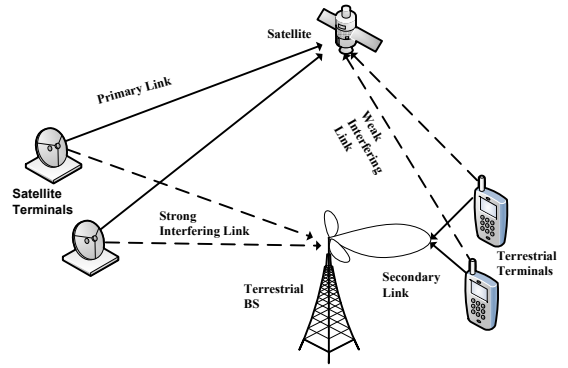

Fig. 1. Hybrid satellite cognitive scenario for multiuser link coexistence

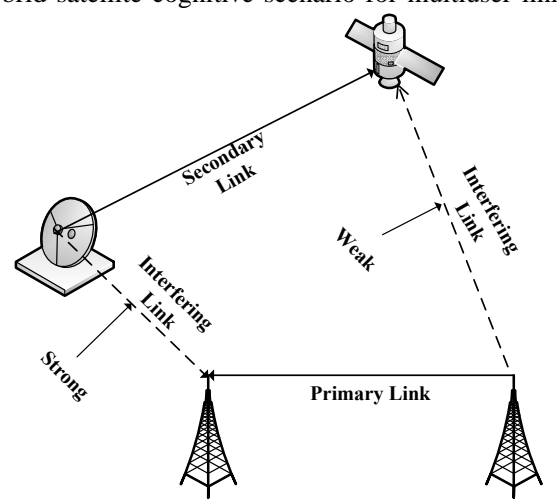

Fig. 2. Hybrid satellite cognitive scenario for single user coexistence

Both uplink and downlink FSS coexistence can be considered with the FS microwave link depending on the frequency of operation. The strong interfering link in this scenario is the link between the satellite terminal and the microwave station. By analyzing the interference between these systems, suitable cognitive techniques can be applied depending on the interference level and allowable level of interaction.

\section{B. Techniques}

1) Spectrum Awareness Techniques: Existing spectrum awareness literature mostly consider exploring spectrum opportunities in the frequency, time and spatial domains. In the context of cognitive SatComs, polarization, radiation pattern can also be considered as additional degrees of freedom for enabling coexistence. From the cognitive point of view, if primary system is designed to transmit in one polarization, secondary system can be designed to transmit in another polarization using the polarization domain as an interference mitigation tool towards the primary systems. Dual polarization plays a significant role in satellite systems to enhance the spectral efficiency since two independent signals can be transmitted on the same spectrum by means of two orthogonal polarizations, hence doubling the available spectrum. The contribution [11] considers the problem of exploiting SS techniques for a dual polarized fading channel in the hybrid cognitive SatComs scenario. The sensing performance of an Energy Detection (ED) based SS technique has been analyzed in a dual polarized fading channel using different combining techniques such as Selection Combining (SC) and Equal Gain Combining (EGC). Starting with the single antenna case, analytical expressions for probability of detection and 
probability of a false alarm have been presented for these techniques in Additive White Gaussian Noise (AWGN), Single Input Multiple Output (SIMO) and Multiple Input Multiple Output (MIMO) Rayleigh fading channels. The simulations as well as theoretical results show that the performance of the ED technique in a MIMO fading channel is much better than in SIMO and SISO fading channels. Furthermore, it has been concluded that the detection performance in a dual polarized fading channel increases with the value of cross polar ratio (XPR) and its lower and upper bounds occur for a SIMO fading channel and an ideal MIMO fading channel respectively. In practical scenarios, blind spectrum awareness techniques need to be investigated since cognitive terminal does not have prior knowledge of PU signal, channel and noise variance. In this direction, the contribution in [22] studies eigenvalue based blind SS techniques and the contribution in [23] investigates the effect of noise correlation on eigenvalue based SS techniques and further purposes eigenvalue based blind SNR estimation technique for estimating the SNR of the PU signal.

2) Interference Modeling: Interference analysis between the satellite and terrestrial links helps to understand the level of interference between these systems while operating within the same spectrum and provides the guidance on the use of suitable cognitive techniques. In this direction, interference modeling between terrestrial BS and the satellite terminal is carried out in [3] on the basis of interference power level. While analyzing the elevation angle range of the points on Europe satellite map coverage for SES ASTRA 2D satellite, it has been observed that it ranges from $7.07^{\circ}$ to $49.40^{\circ}$. It has been observed that satellite terminals near polar region receive more interference from a terrestrial BS than the terminals located near the equator due to the variation in elevation angle. Furthermore, it has been concluded that different cognitive techniques can be considered based on interference power level. SS and DB techniques seem to provide best performance in high interference region and in low or medium interference region, interference from secondary system to primary system can be suppressed by using some form of underlay technique. Although overlay technique can be used in both high interference and low interference scenario by using advanced coding and modulation schemes, it appears to be suitable only for integrated systems with very high level of interaction.

3) Beamforming: Existing spectrum sharing techniques mostly consider three signal dimensions i.e., frequency, time and area for sharing the available spectrum between primary and secondary systems. However, due to advancement in smart antennas and beamforming techniques, multiple users can be multiplexed into the same channel at the same time and in the same geographical area. In this context, angular dimension or directional dimension of the spectral space can be considered as a more efficient way of exploiting the underutilized primary spectrum for the SUs. In the context of considered hybrid cognitive SatComs (Fig. 2), contributions [12] and [13] study transmit and receive beamforming techniques using the unique transmission characteristics of GEO satellite terminals

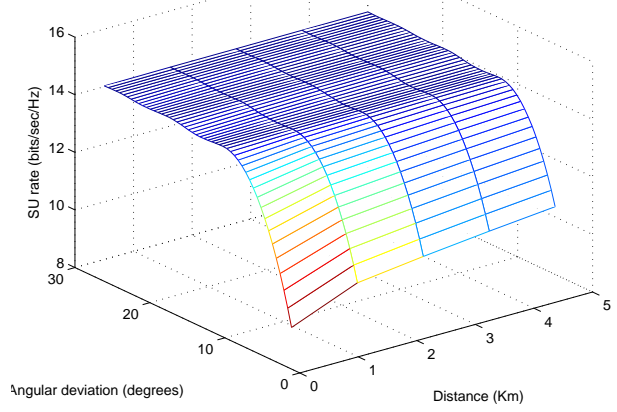

Fig. 3. Worst case SU rate versus PU distance and angular deviation from the sector of interest

respectively. GEO satellites are located in the geosynchronous orbit above the equator and therefore transmit in a northerly direction if we consider the European continent. The GEO satellite terminals have therefore the special propagation characteristic to always point towards the GEO satellites (south). While considering the coexistence of a satellite network with the terrestrial cellular network, the interference received by the BS is concentrated in a specific angular sector. This priori knowledge has been considered in [13] in order to investigate different receive beamforming techniques such as Linear Constrained Minimum Variance (LCMV) and Minimum Variance Distortionless Response (MVDR) for the considered scenario. Similarly, while considering transmit beamforming scenario, the reception range of all the satellite terminals is concentrated in an angular sector. This priori knowledge has been considered in [12] in order to investigate different transmit beamforming techniques such as scaled LCMV, modified LCMV and rate maximization technique for the considered scenario. Figure 3 shows the worst case SU rate versus PU distance and the angular deviation from the considered interfering sector using the rate optimization technique considered in [12]. Furthermore, it has been shown that the SU rate also depends on the constrained power thresholds in the direction of the PUs.

\section{Dual Cognitive SatComs}

\section{A. Scenarios}

The cognitive dual systems can be broadly categorized into (i) Two Geostationary Earth Orbit (GEO) satellite coexistence (Fig. 4), and (ii) GEO and Low Earth Orbit (LEO)/ Medium Earth Orbit (MEO) satellite coexistence (Fig. 5). The frequency of interest for this scenario is Ka-band. The primary system is the existing GEO satellite and the secondary can be another GEO or LEO/MEO satellites. In two GEO coexistence scenario, cognitive techniques may differ for the coexistence of two multibeam systems and for the coexistence of multibeam and monobeam systems. Another scenario of GEO and LEO/MEO coexistence scenario has also received important practical consideration due to deployment of different LEO/MEO satellites within the same spectrum to improve the satellite transmission latency. 


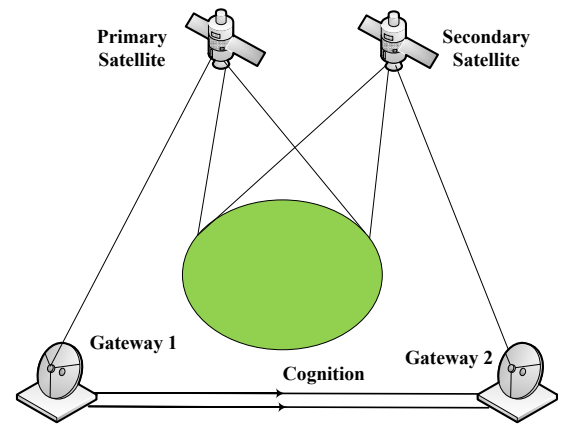

Fig. 4. Dual satellite cognitive scenario for two GEO coexistence

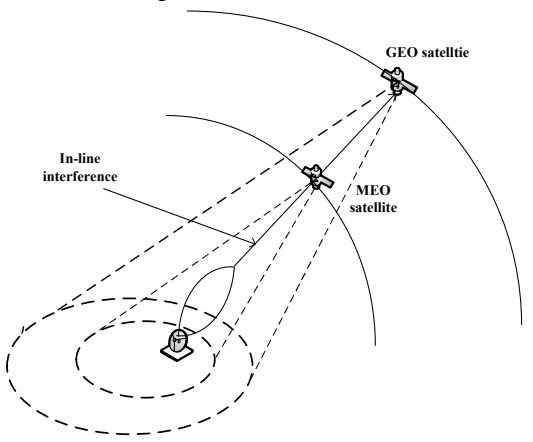

Fig. 5. Dual satellite scenario for GEO and MEO/LEO coexistence

\section{B. Techniques}

1) Spectrum Awareness Techniques: Dynamic spectrum awareness techniques can also be applied for dual satellite coexistence scenario provided that the cognitive satellite terminals are equipped with suitable RF front-ends. The main difficulty in this case lies in acquiring wideband satellite signal. However, instead of detecting the entire signal, some of the characteristics of the primary signal such as sparsity order, SNR, polarization can be acquired to enable the coexistence. In [14], the problem of enhancing SS efficiency has been considered for the considered dual cognitive scenario (Fig. 4) exploiting the polarization domain. By deploying two orthogonally polarized antennas, any type of receive or transmit polarization can be derived. Detecting the polarization state in addition to energy of a certain carrier frequency can significantly enhance the spectrum efficiency. In this direction, different combining techniques for SS have been studied in [14] considering a dual polarized AWGN channel. Furthermore, polarization states of the received signals have been exploited and based on obtained polarization states, Optimum Polarization Based Combining (OPBC) technique has been proposed for carrying out SS in the satellite terminals equipped with dual polarized antennas. The sensing performance of the proposed OPBC technique has been compared to SC, EGC and Maximum Ratio Combining (MRC) techniques. With the help of simulation results, it has been shown that the OPBC technique achieves a significant improvement in sensing performance over other techniques at the expense of complexity.

2) Interference Alignment: IA is an interference mitigation technique and can be used as an underlay technique for enabling the coexistence of wireless networks. This technique has received important attention recently in the CR research community [16], [24]. In the context of dual satellite systems, the contribution [16] investigates an underlay spectral coexistence mechanism exploiting the uplink IA in order to mitigate the interference of multibeam satellite terminals towards the monobeam satellite. Three different types of IA techniques, namely, coordinated, uncoordinated and static, have been investigated depending on the level of network interaction and adaptation with the channel conditions. The performance of these techniques is evaluated and compared with the primary only, resource division and no-mitigation techniques in terms of ergodic sum-rate capacity, primary rate protection ratio and primary to secondary rate ratio. It has been noted that the coordinated IA technique achieves the highest sum-rate while providing perfect protection to the primary system. To enhance the spectrum efficiency further, the contribution [18] investigates the frequency packing technique for IA based dual satellite cognitive systems.

3) Cognitive Beamhopping: A beamhopping satellite system can operate by using full frequency reuse over a certain beamhopping pattern. The main difference from conventional multibeam systems is that multiple beams within a cluster share available spectrum in the time domain instead of the frequency domain in conventional multibeam systems. Since the primary satellite only illuminates a small fraction of beams out of a large number of beams deployed under beamhopping systems, the rest of the beams remain idle at that time waiting for their transmission slots. In this context, the overall system spectrum efficiency can be enhanced if we could deploy a secondary satellite within the same spectrum without producing harmful interference to the primary system. In this direction, the contribution in [17] proposes a cognitive beamhopping system with the objective of enhancing the system spectral efficiency while protecting the primary system. The primary satellite is assumed to be operated with larger beams and the secondary satellite with smaller beams in the same coverage area. The cognition can be achieved by sharing the beamhopping pattern and the timing information of the primary multibeam system to the secondary multibeam system using a signalling link between their corresponding gateways. The performance of the proposed cognitive beamhopping system has been evaluated and compared with the conventional multibeam and beamhopping systems. It is concluded that the cognitive beamhopping system significantly improves the spectral efficiency over other techniques as reflected in Fig. 6.

\section{OPEn Issues}

There exists several technical, regulatory and business challenges for effective implementation of cognitive SatComs. We provide some open research issues from the technical perspective below.

- Interference modeling using a unified framework by considering the aggregate secondary network interference and analyzing its impact on the primary network in the context of cognitive SatComs is a relevant research issue. 


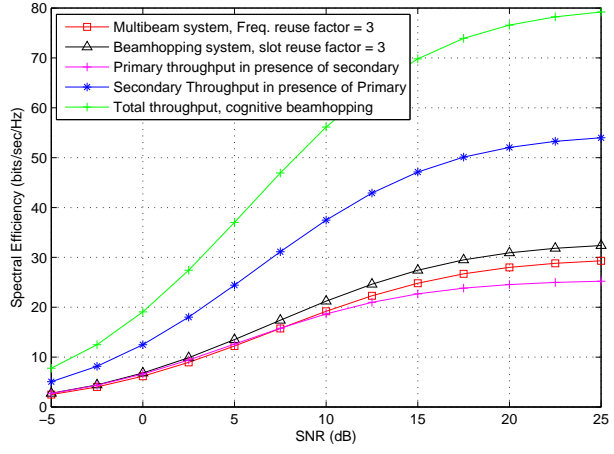

Fig. 6. Throughput comparison of different systems (Reuse factor $(\mathrm{K})=3$, Number of beams $\left(N_{\mathrm{b}}\right)=19$, Secondary power $\left(P_{\mathrm{st}}\right)=$ Primary power $\left.\left(P_{\mathrm{pt}}\right)=10 \mathrm{dBW}\right)$

- Most of the existing contributions consider the coexistence of a single primary system with a single secondary system. However, in practical scenarios, several secondary systems need to coexist with many primary systems. In this aspect, identifying feasible coexistence techniques for allowing the coexistence of multiple primary and secondary systems is another aspect to be addressed.

- Investigating wideband blind sensing techniques for identifying temporal spectrum holes in the satellite spectrum.

- Exploring the possibility of using cognitive techniques in all four types of modes mentioned in Section II-A for implementing cognitive SatComs effectively.

- Developing less complex resource allocation methods for hybrid/dual cognitive SatCom systems.

- In GEO and LEO/MEO coexistence scenario, investigating suitable cognitive technique in order to protect the already existing GEO systems from LEO/MEO interference with a smaller constellation size is a relevant research problem.

\section{CONCLUSIONS}

Cognitive SatComs can be a promising technology for enabling the coexistence of future hybrid/dual satellite networks. In this paper, possible hybrid/dual satellite cognitive coexistence scenarios have been presented and several cognitive techniques have been identified for enabling these scenarios. Furthermore, an overview of these techniques have been included by referring to the existing literature and several future research issues have been identified in this domain.

\section{ACKNOWLEDGEMENT}

This work was supported by the National Research Fund, Luxembourg under AFR (Aids Training-Research) grant for PhD project (Reference 3069102) and the CORE project "CO2SAT: Cooperative and Cognitive Architectures for Satellite Networks".

\section{REFERENCES}

[1] K. Patil, R. Prasad, and K. Skouby, "A survey of worldwide spectrum occupancy measurement campaigns for cognitive radio," in proc. ICDeCom, Feb. 2011, pp. $1-5$.
[2] P. Chini, G. Giambene, and S. Kota, "A survey on mobile satellite systems," Int. J. Satell. Commun. Network, vol. 28, no. 1, pp. 29-57, 2009.

[3] S. K. Sharma, S. Chatzinotas, and B. Ottersten, "Satellite cognitive communications: Interference modeling and techniques selection," in proc. 6th ASMS and 12th SPSC, 2012, pp. 111-118.

[4] D. Ahn, et al, "Integrated/hybrid satellite and terrestrial networks for satellite IMT-advanced services," Int. J. Satell. Commun. Network, 2010.

[5] S. Kota, "Hybrid/integrated networking for NGN services," in proc. Wireless VITAE, 2nd Int. Conf. on, March 2011, pp. 1 -6.

[6] S. Kandeepan, L. De Nardis, M.-G. Di Benedetto, A. Guidotti, and G. Corazza, "Cognitive satellite terrestrial radios," in proc. IEEE GLOBECOM, Dec. 2010, pp. $1-6$.

[7] M. Haddad, M. Debbah, and A. Hayar, "Distributed power allocation for cognitive radio," in proc. 9th ISSPA 2007, Feb. 2007, pp. 1 -4.

[8] E. Del Re, et al, "A power allocation strategy using game theory in cognitive radio networks," in proc. Int. Conf. Game Theory for Networks, May 2009, pp. $117-123$.

[9] A. Panagopoulos, et al, "Coexistence of the broadcasting satellite service with fixed service systems in frequency bands above $10 \mathrm{GHz}$," IEEE Trans. Broadcasting, vol. 52, no. 1, pp. 100 - 107, March 2006.

[10] D.-S. Oh, et al, "A study on the separation distance for frequency sharing between gso network and terrestrial network in Ka band," in proc. IEEE Veh. Techol. Conf., May 2008, pp. $2967-2971$.

[11] S. K. Sharma, S. Chatzinotas, and B. Ottersten, "Spectrum sensing in dual polarized fading channels for cognitive SatComs," in proc. IEEE GLOBECOM, 2012, pp. 3419-3424.

[12] S. K. Sharma, S. Chatzinotas, and B. Ottersten, "Transmit beamforming for spectral coexistence of satellite and terrestrial networks" to appear in proc. 8th Int. Conf. CROWNCOM, July 2013.

[13] S. K. Sharma, S. Chatzinotas, and B. Ottersten, "Spatial filtering for underlay cognitive SatComs," to appear in proc. 5th Int. Conf. PSATS, June 2013.

[14] S. K. Sharma, S. Chatzinotas, and B. Ottersten, "Exploiting polarization for spectrum sensing in cognitive SatComs," in proc. 7th Int. Conf. CROWNCOM, 2012, pp. 36-41.

[15] L. N. Wang and B. Wang, "Distributed power control for cognitive satellite networks," Advanced Materials Research: Mechatronics and Intelligent Materials II, vol. 71, pp. 1156-1160, March 2012.

[16] S. K. Sharma, S. Chatzinotas, and B. Ottersten, "Interference alignment for spectral coexistence of heterogeneous networks," EURASIP J. Wireless Commun. and Networking, vol. 46, 2013.

[17] S. K. Sharma, S. Chatzinotas, and B. Ottersten, "Cognitive beamhopping for spectral coexistence of multibeam satellites," in Future Network Mobile Summit 2013, July 2013.

[18] S. Chatzinotas, S. K. Sharma, and B. Ottersten, "Frequency packing for interference alignment-based cognitive dual satellite systems," to appear in proc. VTC Fall-2013, Sept. 2013.

[19] B. Evans, et al, "Integration of satellite and terrestrial systems in future multimedia communications," IEEE Wireless Commun., vol. 12, no. 5, pp. $72-80$, Oct. 2005 .

[20] S. Kandeepan, L. De Nardis, M.-G. Di Benedetto, A. Guidotti, and G. Corazza, "Cognitive satellite terrestrial radios," in proc. IEEE GLOBECOM, Dec. 2010, pp. 1 -6.

[21] E. Del Re, et al, "Power allocation strategy for cognitive radio terminals," in proc. First Int. Workshop on CR and Advanced Spectrum Management, Feb. 2008, pp. 1 -5.

[22] S. Chatzinotas, S. K. Sharma, and B. Ottersten, "Asymptotic analysis of eigenvalue based blind spectrum sensing techniques," to appear in proc. ICASSP 2013, May 2013.

[23] S. K. Sharma, S. Chatzinotas, and B. Ottersten, "Eigenvalue based sensing and SNR estimation for cognitive radio in presence of noise correlation," IEEE Trans. Veh. Technol., vol. 62, no. 8, pp. 1-14, 2013.

[24] B. Koo, D. Park, "Interference Alignment with Cooperative Primary Receiver in Cognitive Networks," IEEE Communications Letters, vol.16, no.7, pp.1072-1075, July 2012. 